\title{
Analysis Methodology for Flow-level Evaluation of a Hybrid Mobile-Sensor Network
}

\author{
D. C. Dimitrova ${ }^{1}$, G. Heijenk ${ }^{2}$, and T. Braun ${ }^{1}$ \\ 1 Univeristy of Bern, Bern, Switzerland \\ 2 University of Twente, Enschede, The Netherlands
}

\begin{abstract}
Our society uses a large diversity of co-existing wired and wireless networks in order to satisfy its communication needs. A cooperation between these networks can benefit performance, service availability and deployment ease, and leads to the emergence of hybrid networks. This position paper focuses on a hybrid mobile-sensor network identifying potential advantages and challenges of its use and defining feasible applications. The main value of the paper, however, is in the proposed analysis approach to evaluate the performance at the mobile network side given the mixed mobile-sensor traffic. The approach combines packetlevel analysis with modelling of flow-level behaviour and can be applied for the study of various application scenarios. In this paper we consider two applications with distinct traffic models namely multimedia traffic and best-effort traffic.
\end{abstract}

\section{Introduction}

In the last few years a fast development in the area of wireless applications is observed. On the one hand, traditional infrastructure-based cellular networks have undergone major changes with the shift toward UMTS and its successor LTE. On the other hand, wireless networks with local geographical coverage, such as wireless sensor networks, attracted the interest of both industry and academia. Indeed, a survey of the ongoing research efforts by both industry and academia shows that the topic of network interoperability is very attractive, see $[7,11]$. We recognise that both types of networks have merits and propose to combine them in one hybrid mobile-sensor network.

In this paper we are specifically interested in the impact of sensor traffic on the performance of mobile traffic and how the phenomenon can be best studied. To this end we propose an approach that can capture both mobile traffic specifics and Quality of Service (QoS) requirements of sensor traffic. Special attention is paid to the impact of flow dynamics, i.e., the generation of flows at arbitrary moments and arbitrary locations, which leads to a changing number of service requests. In previous studies, see $[3,4]$, it was shown that flow dynamics are crucial for the performance of data traffic. We expect this to hold even stronger when several traffic classes are considered.

A hybrid mobile-sensor network arises many research challenges among which also resource management and QoS provisioning. These two topics have received 
much attention in the literature as $[2,5,8]$ and [12] attest. Although providing much insight the authors, e.g., $[1,6,10,11]$, often consider only one type of network - mobile or sensor. However, in a hybrid network there are more factors that contribute to the complexity of the problem. There are several proposals, e.g., $[7,9]$, which describe a complex new architecture to support the cooperation between wireless networks. Unfortunately, in the general case such studies provide only a highly abstract view without discussing in detail specific network functionality. To the best of our knowledge there are no studies specifically dedicated to the topic of resource management in a hybrid mobile-sensor network, independently of the presence of flow-level analysis.

An interesting study related to our proposal is [7]. The authors evaluate the cooperation between UMTS and WLAN networks with an architecture similar to the one we propose. However, [7] focuses on protocol support issues and not on resource management. Another interesting reference is [10] which addresses QoS provisioning in a LTE network with mixed traffic, e.g. voice, video and data. The authors provide a theoretical as well as a simulation approach to evaluate performance but do not account for the dynamic behaviour of the users and their main focus is on the voice traffic.

We propose to use a combined analysis approach which includes modelling of flow-level behaviour (left out by many studies) and can therefore offer new perspectives on performance. In contrast to other authors we are not interested in developing a new platform for QoS provisioning but consider QoS parameters as factors that set constraints on resource management and hence have an effect on performance. To achieve the latter we intend to use a modified version of the combined analytical/simulation approach proposed in [3]. Advantages of the approach are its ability to capture in sufficient detail both packet and flow-level behaviour while still supporting quick evaluation.

The paper continues as follows. The next Section 2 presents the concept of a hybrid mobile-sensor network and discusses potential applications. In Section 3 we describe the specific research scenario chosen to investigate in this paper. The proposed analysis methodology is explained in Section 4. Finally, the planned future development is given in Section 5 .

\section{Hybrid Mobile-Sensor Network}

The proposed hybrid network is shown in Figure 1 and consists of a sensor and a mobile domain. The mobile domain is typically represented by a single cellular network with its entities - base stations and a core infrastructure. The specific choice of mobile technology in the current study falls on the Long Term Evolution (LTE). The sensor domain can be formed by several wireless sensor networks, which in turn can have sub-networks. In Figure 1 we indicate two connectivity modes between the domains. In the first case each sensor node can communicate directly to a base station. Such deployment is challenging in terms of implementation, i.e., each sensor node needs to be equipped with two wireless interfaces. Besides, not every sensor node might be able to 'see' the 


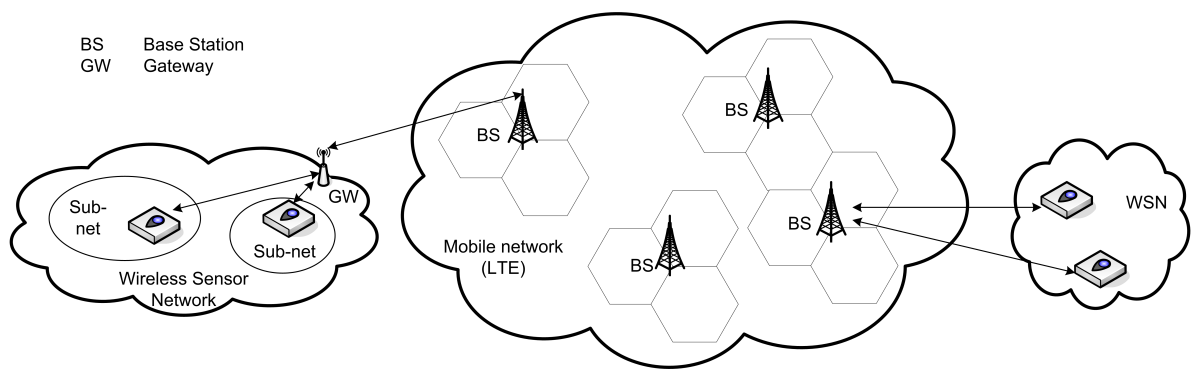

Fig. 1. Hybrid network consisting of sensor and mobile domain.

mobile network, e.g., due to shadowing effects. In the second case the sensor nodes communicate to a dedicated gateway which provides connection to the mobile network. In this approach only a single entity, i.e., the gateway, needs to have two interfaces. The gateway can be positioned either at the sensor network side or at the mobile network side.

\subsection{Deployment Potential}

We consider a hybrid mobile-sensor network interesting for practical deployment due to the individual merits of each participant. A mobile network is characterised by ubiquitous coverage and a reasonably high throughput. Furthermore, base stations generally do not experience energy constraints or buffer shortage contrary to sensor nodes. In terms of scalability, however, sensor networks are cheaper and easier to deploy. They are also versatile in terms of application purposes. Hence, the benefits that a hybrid mobile-sensor network may offer and the challenges that it may pose depend on the taken perspective.

From a sensor network perspective there are multiple expected gains. For example, reporting via a mobile network can positively affect the lifetime of the sensor network. A phenomenon typically observed in sensor networks is the waste of energy by sensor nodes while waiting for the sink to wake up. If a dedicated gateway, constantly powered electrically and always awake, is used as a sink a sensor node can send to it directly, thus decreasing energy consumption. Further, the much larger buffer capacity of the mobile network can offer an excellent temporal storage solution to the sensor network. However, the reverse situation - the mobile network wants to send (large) data to the sensor network this may lead to problems such as buffer overflow and increased collisions in the wireless 'sensor' channel.

From a mobile network perspective there are arguably fewer benefits from the cooperation with a sensor network, partly explained by the technical superiority of the mobile network. The potential opportunities however are worth exploring. For example, a mobile network can use the sensor network to perform measurements, which can then be offered to interested third parties. Sensor nodes can be deployed easily and quickly, which can be used by mobile operators for 
the monitoring and maintenance of mobile network sites. There are few challenges as well, e.g., support of traffic differentiation and priority service. These required changes, however, can benefit the service of mobile traffic as well, e.g., to guarantee the QoS requirements of voice calls or to offer preferential service to emergency traffic. Note that LTE is developed for packet-switched traffic only which drives researchers to investigate possibilities for QoS support, see $[1,10]$

\subsection{Application Scenarios}

We identify the following big groups of possible applications for a hybrid mobilesensor network - monitoring, streaming, event detection and remote activation.

Monitoring Wireless sensor networks (WSNs) can be used, e.g., for environmental monitoring, health monitoring, construction monitoring or weather forecasting. Such applications are associated with periodic transmission of the collected sensor data. Still, the frequency of reporting may differ from every few minutes to every few hours or even days and depends on the specific application. For example, a health monitoring system needs to report every few minutes in order to ensure the safety of the patient. A sensor network that collects data for weather forecasting, however, may aggregate the data and report only once a day. Independently of the scenario a mobile network can be used to temporally store and deliver the collected sensor data to a central collection point.

Streaming Monitoring for security or safety purposes generates continuous stream of data over long periods of time and we give it in an individual category. Streaming applications, in contrast to monitoring, is characterised by long lifetimes and larger data traffic. Some examples of streaming are video surveillance of restricted areas or areas with high crime risk, video surveillance for traffic control purposes or remote audio surveillance. Such applications generate multimedia type of traffic, e.g., voice, audio, video, with strict (and divers) QoS requirements and can therefore benefit significantly from the high bandwidth capacity of a mobile network.

Event detection WSNs can be also used to detect the occurrence of an event. In this more passive mode a senor node is typically asleep and awakes only in the occasion of a specific event. Upon event detection multiple sensors transmit data at the same time. The total amount of generated sensor data depends on the number of deployed sensor nodes and the type of measurements and can be quite bursty as well. If only an alarm needs to be activated, e.g., in case of fire detection, the data is much less compared to data gathered during a parameter reading, e.g., in case of landscape profiling by echo sounding.

Remote activation It is possible that the mobile network wants to communicate to all nodes in a particular sensor network, e.g., to remotely (re-)configure the sensor network, to disseminate software updates or to request measurements by the sensors. The latter can be used to acquire information on channel fading at a specific frequency by measuring the received signal of each sensor and comparing it to the transmitter metrics that the sensor reported. 
In order to illustrate the potential of the proposed hybrid network we describe few feasible applications but a hybrid network can be deployed with success in many more situations. Our first example is a health monitoring application. A health monitoring unit (consistent of sensors) allows high-risk patients to have normal life while still providing an appropriate medical supervision. A health monitoring system should be at all times connected to a central server that can alert medical personnel if necessary. If the patient is on the move a mobile network can provide uninterrupted connectivity.

In the second example we consider a disaster situation involving a building collapse. A wireless sensor network is easily deployed and can provide local emergency services with valuable information. By connecting to a mobile network the collected sensor data can be further distributed towards experts at remote locations. If the local mobile network infrastructure was fully or partially destroyed other intermediate solutions, e.g. a mesh network, can be deployed to bridge communication.

Finally, an interesting example is a habitat monitoring application. Despite being used for monitoring purposes it is in fact an event detection - the sensors are activated by an approaching animal. If a mobile network is in range the data can be directly relayed to a central database enabling the more bufferchallenging video monitoring. Note however that energy consumption is still an issue. If mobile coverage is scares, e.g., in large isolated areas, observation is kept to the basic data collection.

\section{$3 \quad$ Research Topic}

Before a hybrid mobile-sensor network can be deployed there are several challenges that need to be addressed; some were already mentioned, e.g., buffer management, load balancing and QoS provisioning, others include network discovery, radio resource control, signalling and sensor data protection. In this study we are only interested in the challenges related to radio resource management (and scheduling in particular) in the mobile network. Note that resource management in the mobile and in the sensor network domains are two separate issues with network-specific challenges. Mobile network scheduling is affected by mainly two aspects of the sensor network namely the traffic size and the QoS requirements both determined by the application type and the deployment scenario. QoS requirements, e.g. delays, throughput, packet loss, of a sensor network traffic can vary significantly; for example health monitoring data is critical and should be served as first at all times while weather forecasting data only needs guaranteed delivery.

In the current study we focus on a streaming scenario and an event detection scenario with large data traffic. The two scenarios differ significantly in terms of traffic characteristics, including QoS parameters, which is why we chose them. In the streaming scenario the sensor nodes are distributed over a large geographical area, e.g., an industrial area. Each sensor connects via a gateway to the closest base station from where the sensor data is propagated to a central database. It 


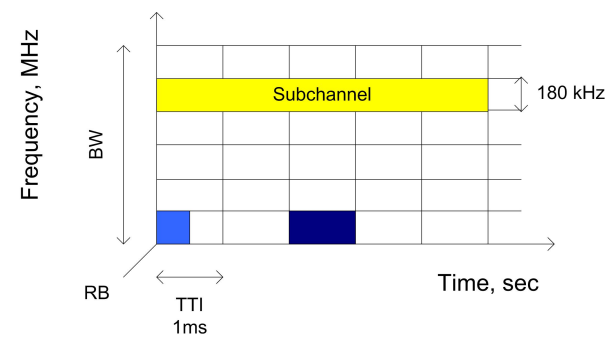

Fig. 2. LTE radio resource structure.

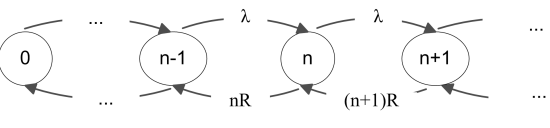

Fig. 3. Markov model of a single cell with mobile traffic only.

is assumed that the sensors are connected to the power grid and hence energy consumption is not an issue. Streaming applications generate a continuous traffic stream which is sensitive to transmission delays. Therefore, they would require to be served with highest priority.

For the event detection scenario we are interested in a situation where a large number of sensors is spread over an area of limited size and each sensor collects large amounts of data. An example of such an application is landscape profiling by using echo sounding. Both the number of sensors and the amount of sensor data cause a significant increase in traffic for the mobile network. The data is bursty and of best effort type, i.e., it can tolerate delays as long as the data is correctly transmitted.

In both scenarios the mobile network perspective is considered, i.e., what changes are observed in the mobile network performance after the introduction of sensor network traffic. The choice of LTE as the mobile technology has the advantage of possibility to schedule in the time and frequency domain. This offers flexibility to meeting the various traffic demands and QoS requirements, associated with the streaming and event detection scenario. The possibility to schedule in the frequency domain may also provide easy means to separate mobile from sensor traffic handling.

In order to investigate how the performance of mobile users is affected by the introduction of sensor network traffic we need an approach that can capture both the specifics of the data traffic in the mobile networks as well as the specifics of the sensor network traffic. Further, we argue that it is important to take into account the change in number of ongoing flows in both networks; this is especially important for the streaming application. The proposed methodology is further described in the following Section 4.

\section{Analysis Methodology}

In order to enable performance evaluation in the mobile network for both scenarios, streaming and event detection, we propose a modified version of the analysis approach proposed in [3]. The basic form of the approach, which combines system analysis at packet level with modelling of user behaviour at flow level, is 
presented in Section 4.1. Later, in Section 4.2 we discuss the modifications that each scenario needs, due to specific QoS requirements.

\subsection{Basic Approach}

The system analysis at packet level captures the impact of several factors among which scheduler specifics (e.g., resource size), environmental characteristics (e.g., interference) and user specifics (e.g., power headroom). The performance of a mobile user is characterised by an instantaneous rate $r$ and a state-dependent throughput $R$. In order to explain the differences we need to refer to the radio resource management in LTE. Recall that in LTE scheduling in two dimensions is possible, see Figure 2. In the time dimension access in organised in Transmission Time Intervals (TTIs) of $1 \mathrm{~ms}$ while in the frequency dimension the system bandwidth is divided over 'sub-channels' of $180 \mathrm{kHz}$. The intersection of a TTI and a sub-channel defines the smallest radio resource unit that can be allocated to a user.

The instantaneous rate $r$ is the data rate realised by a user within a TTI and it is calculated over all resource units allocated to that user. It is determined by the signal-to-interference-plus-noise ratio(SINR), the possible modulation and coding schemes (MCS) and the receiver characteristics related to that MCS. If the user was not scheduled for service in the TTI intuitively $r=0$. The statedependent throughput $R$ captures the impact of the number of ongoing flows $n$ on the service, i.e., the instantaneous rate, of a particular user. Referring back to Figure 2, depending on the allocation strategy and the number of users $n$ it may happen that several TTIs are needed to serve all requests. This effect is captured by $R$, which is derived as $R=r / c$ where $c$ is the transmission cycle, i.e., the total number of TTIs necessary to serve all users at least once.

The dynamic behaviour of the system at flow level is modelled by continuous time Markov chains. They are very appropriate for the task since a state in the chain can be mapped to a state in the system, i.e., the number of ongoing flows $n$. In Figure 3 we give an example for a single cell. Each state in the Markov chain corresponds to a number of currently active users in the cell. The jumps in the Markov chain represent the initiation and completion of flow transfers. The corresponding transition rates (for a particular state) are determined from the (a-priori) given user arrival rate $\lambda$ and the state-dependent throughput $R$ (in that state).

Eventually, the evolution of the number of mobile data users corresponds to a sequence of state transitions in the Markov chain. From the steady-state distribution of this Markov chain we can derive performance parameters, e.g., (long-term) flow throughput or fairness index. The steady-state distribution of the Markov chain can be found, in special cases, by analytical approaches leading to explicit closed-form expressions. When the resulting form of the Markov chain is very complex and not trivial to solve, simulation of the state transitions can provide the means to derive the steady state distribution. 
Working with Markov models has many advantages among which time-efficient performance evaluation, scalability in the system size and easy parameter modification due to the division of packet and flow level analysis.

\subsection{Modified Approach}

The introduction of sensor traffic to the analysis requires different changes to be made depending on whether the streaming or the event detection scenario is addressed. As previously discussed streaming applications need to be served with highest priority meaning that part of the radio resource is exclusively reserved for the sensor traffic. The size of the reservation depends on the number of active sensor nodes and on the multimedia class, i.e., audio, video, and determines how much resource is left for the service of the mobile users. In the event detection scenario however no priority service is required and sensor traffic is treated equally with mobile data traffic. All sensor data generated at a particular moment can be managed as a single data flow.

For both scenarios we do not foresee explicit changes of the expressions for the instantaneous rate and the state-dependent throughput since the effects are hidden in the calculation of the available bandwidth. The form of the Markov chain, however, needs to be adapted in order to capture the behaviour of sensor traffic. For the streaming scenario we need to add a new dimension, which represents the changes in the number of multimedia flows, while in the event detection scenario a new dimension with only two states - no sensor data and data - suffice.

The proposed methodology can be also easily applied to study a mobile network with monitoring traffic. In our opinion a monitoring scenario is less interesting since: (i) monitoring generates traffic at a predefined time interval, generally bigger than a minute; and (ii) monitoring data is of relatively small size. As result we did not expect a big impact on the performance of an LTE mobile network, given its large bandwidth capacity and scheduling periods of $1 \mathrm{~ms}$.

\section{$5 \quad$ Future Steps}

As a next step in the research we will systematically work out the analysis approach for the streaming and event detection scenarios. This will provide us with the corresponding Markov chains and will allow us to observe performance in the mobile network for various evaluation scenarios, e.g., video only traffic, combined video and audio traffic, mobile data traffic only (reference case).

\section{Acknowledgements}

We want to thank Nirvana Meratnia (University of Twente) for her feedback on the application scenarios and Markus Anwander and Philipp Hurni (University of Bern) for the insights on deployment issues in wireless sensor networks. 


\section{References}

1. M. Anas, C. Rosa, F. D. Calabrese, K. I. Pedersen, and P. E. Mogensen. Combined admission control and scheduling for QoS differentiation in LTE uplink. In VTCFall 2008, IEEE Computer Society Press, pages 1 -5, 2008.

2. D. Chen and P. Varshney. QoS support in wireless sensor networks: a survey. In International Conference on Wireless Networks, 2004.

3. D. C. Dimitrova. Analysing uplink scheduling in mobile networks. A flow-level perspective. PhD thesis, Wöhrmann Print Service, 2010.

4. D.C. Dimitrova, J.L. van den Berg, and G. Heijenk. Performance of relay-enabled uplink in cellular networks - a flow level analysis. In International Conference on Ultra Modern Telecommunications, IEEE Computer Society Press, pages 1-8, 2009 .

5. H. Ekstrom. QoS control in the 3GPP evolved packet system. Communications Magazine, IEEE, 47(2):76 -83, 2009.

6. T. J. Harrold and A. R. Nix. Capacity enhancement using intelligent relaying for future personal communication systems. In VTC-Fall 2000, IEEE Computer Society press., pages $2115-2120$.

7. M. Jacobsson, J. Hoebeke, S. Heemstra-de Groot, A. Lo, I. Moerman, I. Niemegeers, L. Munoz, M. Alutoin, W. Louati, and D. Zeglache. A network architecture for personal networks. In 14th IST Mobile and Wireless Communications Summit, 2005.

8. Y. Li, C. S. Chen, Y-Q Song, and Z. Wang. Real-time QoS support in wireless sensor networks: a survey. In 7th IFAC International Conference on Fieldbuses 86 Networks in Industrial $\&$ Embedded Systems, 2007.

9. E. de Poorter, B. Latr, I. Moerman, and P. Demeester. Symbiotic networks: Towards a new level of cooperation between wireless networks. In Wireless Personal Communicaitons, Springer Netherlands., pages 479-495, 2008.

10. I. Siomina and S. Wanstedt. The impact of qos support on the end user satisfaction in lte networks with mixed traffic. In PIMRC 2008, IEEE Computer Science press, pages $1-5,2008$.

11. H. Y. Wei, S. Ganguly, and R. Izmailov. Ad hoc relay network planning for improving cellular data coverage. In PIMRC 2004, IEEE Computer Science press, pages 769-773, 2004.

12. F. Xia. Qos challenges and opportunities in wireless sensor/actuator networks. Sensors, 8(2):1099-1110, 2008. 